\title{
SANS studies of nanostructured low-melting metals at room temperature
}

\author{
A. A. Naberezhnov ${ }^{1}$, S. A. Borisov ${ }^{1}$, A. V. Fokin ${ }^{1}$, A. Kh. Islamov ${ }^{2}$, A. I. Kuklin ${ }^{2}$, Yu. A. Kumzerov ${ }^{1}$ \\ ${ }^{1}$ Ioffe Institute, Polytekhnicheskaya 26, St. Petersburg, 194021, Russia \\ ${ }^{2}$ Frank Laboratory of Neutron Physics, Joint Institute for Nuclear Research, 141980 Dubna, Russia \\ alex.naberezhnov@mail.ioffe.ru, sergey.borisov@mail.ioffe.ru, Midbarzin@yandex.ru, \\ akhmed.islamov@gmail.com, alexander.iv.kuklin@gmail.com
}

\begin{abstract}
Nanocomposite materials (NCM) based on micro- and macroporous glasses containing nanoparticles of In, Sn and $\mathrm{Pb}$ into porous space have been studied by small-angle neutron scattering (SANS) at room temperature. The dependencies of fractal characteristics of metals embedded into the pores from the value of transferred impulse $Q$ have been obtained. The existence of a critical spatial scale ( $15-16 \mathrm{~nm})$ has been established, at which a change in the fractal characteristics of embedded metals takes plays. Distributions of pair correlation functions have been calculated for all types of the studied NCM. It is shown that in these NCM metals form the complicated space systems combining the crystalline and amorphous states of embedded metals.
\end{abstract}

Keywords: nanocomposites, small angle neutron scattering, porous matrices, fractal structures, low-melting metals.

Received: 31 October 2020

Revised: 3 November 2020

\section{Introduction}

It is well known that the macroscopic physical properties of metals under so-called "restricted geometry" conditions are essentially different from those of the bulk. For example, indium and gallium nanoparticles crystalline phases have been discovered that do not exist for bulk material metals [1-4]. Confinement changes the phonon spectra of metal nanoparticles [5-10] and the properties of their electron subsystems [11-14]. The most surprising changes have been observed for the superconducting properties of gallium, indium, lead and tin [15-20] nanoparticles obtained by introducing these metals into nanoporous alkali borosilicate glasses. Furthermore, for this type of porous glass, we will use the abbreviation PG. The special features of PG are the very flexible geometric form (i.e., as beads, rods, fibers, ultrathin membranes, flat plates) and the controllable average pore diameters in the broad range between 0.3 and $1000 \mathrm{~nm}$ [21]. Special heat treatment of initial alkali borosilicate glasses of a suitable composition between 500 and $700{ }^{\circ} \mathrm{C}$ initiates a phase separation and two different interconnected phases are obtained. The first one is an alkali-rich borate phase soluble in hot mineral acids, water or alcohols. The second one is almost pure silica. This procedure is used practically for the preparation of the commercial controlled-pore glasses (CPG) [22].

Pores in these glasses form through a multiply-connected system of interconnected channels. Porous glasses with a skeleton consisting almost entirely of amorphous silica $(\sim 96 \%)$ are obtained after the leaching procedure. Currently, these glasses are widely used to create nanocomposite materials (NCM). In particular, for porous glasses with an average pore diameter of $7 \mathrm{~nm}$ (PG7), it has been shown that nanocomposites "Sn (or Pb) + porous glass" become the superconductors of the second type, and the critical magnetic fields leading to the destruction of the superconducting state in these composites increase by 60-100-fold [19,20]. The crystal structure of low-melting metals ( $\mathrm{Hg}, \mathrm{Ga}, \mathrm{In}, \mathrm{Sn}$ and $\mathrm{Pb}$ ) embedded into PG7 has been studied by X-ray and neutron diffraction [1, 4, 10, 17, 23-25] and it has been found that these metals form into the pores of PG7 the nanoclusters with characteristic size slightly larger than the average pore diameter. It means that nanoparticles occupy space in several neighboring pores. In spite of the large amount of research devoted to studies of crystal and macroscopic properties of NCM with embedded low-melting metals the peculiarities of internal space organization of nanoparticles and fractal characteristics in these NCM remain unclear. To obtain such data, one of the most informative techniques is the method of small-angle neutron scattering (SANS), and in this paper, we have used this approach for studies of internal structure and fractal characteristics of low-melting metals (In, Sn and Pb) embedded into micro- (PG7) and macroporous (PG-MAP) alkali borosilicate glasses. 


\section{Samples and method}

For sample preparation we used two types of PG: PG7 with average pore diameter $7 \pm 1 \mathrm{~nm}$ and macroporous glasses (PG-MAP) with a broad distribution of pore diameters from $20 \mathrm{~nm}$ up to $\sim 100 \mathrm{~nm}$ (distribution median was about $40-50 \mathrm{~nm})$. Metals in the porous glasses were introduced from a melt at a pressure of $\sim 6 \mathrm{kbar}(\mathrm{lead})$ and $\sim 10-11 \mathrm{kbar}$ (indium, tin). After cooling a melt, the samples were removed, and their surfaces were thoroughly cleaned from the remnants of the bulk material. We prepared four NCM: S1 - In + PG7, S2 - In + PG-MAP, S3 $\mathrm{Sn}+\mathrm{PG}-\mathrm{MAP}, \mathrm{S} 4-\mathrm{Pb}+\mathrm{PG}-\mathrm{MAP}$. S0 was the reference sample of an initial alkali borosilicate glass after a thermal treatment with the composition $96 \% \mathrm{SiO}_{2}-3.8 \% \mathrm{~B}_{2} \mathrm{O}_{3}-0.2 \% \mathrm{Na}_{2} \mathrm{O}$, which has been determined by chemical analysis. This composition was used for the preparation of porous glasses PG7 and PG-MAP.

Small-angle neutron scattering (SANS) experiments were performed on YuMO time-of-flight spectrometer at high flux pulse IBR-2 reactor (JINR, Dubna, Russia) [26, 27]. YuMO is an instrument with two movable detectors system placed at sample-to-detectors distances of 5.28 and $13.4 \mathrm{~m}$, resulting in a $Q$ range of $0.006-0.5 \mathrm{~A}^{-1}$, where $Q=(4 \pi / \lambda) \sin (\theta / 2)$ is a transferred momentum and $\theta$ is the scattering angle. The diameter of the sample in the beam was $14 \mathrm{~mm}$. The measured neutron scattering spectra were corrected for the transmission and thickness of the sample, background scattering on the film substrate and on the vanadium reference sample using SAS software [28, 29], yielding a neutron scattering intensity in absolute units of $\mathrm{cm}^{-1}$. All measurements were carried out at room temperature. The errors in determination of the alpha parameter for all samples (excepting the initial glass S0) on Fig. 2 in the dependences $I(Q) \sim Q^{-\alpha}$ did not exceed $2-3 \%$. For $\mathrm{S} 0$ the error was about $7 \%$.

\section{Results}

Low resolution the SANS technique cannot give the detailed structure at the atomic level but is useful to describe structural features typical at distances of 1 to $100 \mathrm{~nm}$.

In the case of disordered systems, such as glasses with embedded metals, in the absence of strong correlations between inhomogeneities (fluctuations in the scattering density), the scattered intensity can follow the power law of $Q$ :

$$
I(Q)=A \cdot Q^{-\alpha}+B
$$

where $A$ and $B$ are constants, $B$ is the background. It has been shown [30] that for objects with a complex branched surfaces (surface fractal of dimension from $2 \leq D s \leq 3$ ) $3<\alpha \leq 4$ scattering can be given by:

$$
\begin{gathered}
I(Q)=A \cdot Q^{-(6-D s)}+B, \\
A=\pi N_{0}(\Delta \rho)^{2} \Gamma(5-D s) \sin (\pi(D s-1) / 2),
\end{gathered}
$$

where $N_{0}$ is the measure of the fractal surface (in other words, a constant characteristic of the fractal boundaries), $\Gamma-$ gamma function and $\Delta \rho$ (contrast) is the difference between coherent scattering densities of the inner part of cluster and its boundary (surface) layer, which have different composition of elements or lower density packing. In the ideal case, where the interfacial region is sharp, one obtains the equation of Porod's law [31]:

$$
I(Q)=2 \pi(\Delta \rho)^{2}\left(\frac{S}{V}\right) Q^{-4}
$$

where $S / V$ is the total area of the interface per unit of volume of the particle.

From the equation (4), it is easy to see that the parameter $\Delta \rho$ plays the principle role in a dependence $I$ vs. $Q$. The scattering densities of initial glasses with composition $96 \% \mathrm{SiO}_{2}-3.8 \% \mathrm{~B}_{2} \mathrm{O}_{3}-0.2 \% \mathrm{Na}_{2} \mathrm{O}(\mathrm{mass}$. $\%$ ) and metals embedded into porous glasses have been calculated using the program SLD [32] and are presented in Table 1.

TABLE 1. Densities of coherent scattering for glass and metals

\begin{tabular}{|c|c|c|c|c|c|}
\hline Element & $96 \% \mathrm{SiO}_{2}-3.8 \% \mathrm{~B}_{2} \mathrm{O}_{3}-0.2 \% \mathrm{Na}_{2} \mathrm{O}$ & $\mathrm{In}$ & $\mathrm{Sn}$ & $\mathrm{Pb}$ & Air into the pores \\
\hline$\rho_{\text {scatt }}, 10^{10} \mathrm{~cm}^{-2}$ & 3.32 & 1.56 & 2.31 & 3.1 & $\sim 0$ \\
\hline
\end{tabular}

As has been mentioned above, the filling of porous glasses by metals has been performed from the melts. It is natural to expect that due to metal contraction in the pores at cooling, the voids are formed into porous space of glasses. Thus, in these nanocomposites, we have three types of objects (glass skeleton, metal nanoparticles and empty space) and three types of interfaces ("matrix-voids", "matrix - metal nanoparticles" and "voids - metal nanoparticles"). Since the intensity of small-angle scattering into a zero angle is proportional to the square of the contrast $\left((\Delta \rho)^{2}\right)$, the intensity of scattering with a complete filling of the pores with metal would be much less than for empty porous glass (see Table 1 and the equation (5)): 


$$
I(0)=\varphi_{\text {Met }}\left(\rho_{\text {Glass }}-\rho_{\text {Met }}\right)^{2} V,
$$

where $\rho_{\text {Glass }}$ and $\rho_{\text {Met }}$ are the scattering densities of glass and embedded metal. $V$ and $\varphi$ are the volume and the volume fraction of metal nanoclusters. However, the experimental results show (Fig. 1) that scattering intensities from glasses filled by metals significantly exceeds the scattering from the glass PG7. This means the following: $1-\mathrm{S} 0$ is not really porous glass, 2 - in our samples S1 - S4 three types of scattering objects and interfaces exist and one of them is the interface "glass - empty porous space", which increases the total scattering according to the contrast $(\Delta \rho)^{2}$ (Table 1$)$. The volume fractions of nanoclusters and voids in glass can be obtained using the Porod invariant of two and three phase systems, calculated from the equations (6) and (7) [34]:

$$
\begin{gathered}
2 \pi \rho_{\text {Glass }}^{2} \varphi_{\text {pores }}\left(1-\varphi_{\text {pores }}\right)=\int_{0}^{\infty} I(Q) Q^{2} d Q, \\
2 \pi\left\lfloor\rho_{\text {Glass }}^{2} \varphi_{\text {pores }} \varphi_{\text {Glass }}+\rho_{\text {Met }}^{2} \varphi_{\text {pores }} \varphi_{\text {Met }}+\left(\rho_{\text {Glass }}-\rho_{\text {Met }}\right)^{2} \varphi_{\text {Met }} \varphi_{\text {Glass }}\right\rfloor=\int_{0}^{\infty} I(Q) Q^{2} d Q,
\end{gathered}
$$

where $\varphi_{\text {pores }}, \varphi_{\text {Glass }}$ and $\varphi_{\text {Met }}-$ relative volume fractions of pores, glass skeleton and metals in these NCM. And from the additional equations $(8,9)$ connecting the volume fractions of metals and voids with the mass densities of glasses $\rho^{G L}\left(\mathrm{~g} / \mathrm{cm}^{3}\right)$ obtained from gravimetric measurements:

$$
\begin{gathered}
\rho_{\mathrm{SiO}_{2}} \varphi_{\mathrm{Glass}}+\rho_{\mathrm{Met}} \varphi_{\mathrm{Met}}=\rho^{G l}=\frac{M^{G l}}{V^{G l}}, \\
\varphi_{\text {Glass }}+\varphi_{\text {Met }}+\varphi_{\text {Pores }}=1 .
\end{gathered}
$$

\subsection{Initial alkali borosilicate glass $\mathrm{SO}$}

For our initial glass S0 we have observed the principle difference between this glass and the conventional porous glasses [35-38], i.e. in S0 there is no a correlation peak at $Q_{0}=0.015-0.022 \AA^{-1}$ (Fig. 2A). It is shown that this peak is characteristic of the presence of structural units with average size $L \sim 2 \pi / Q_{0}$ and has been earlier observed in papers [35-38] devoted to studies of porous glasses. Since $L$ is essentially larger than the average pore diameter, one can conclude that these units (scatterers) are massive and refer to the internal structure of porous glasses. The origin of this phenomena it connected with a procedure of porous glass preparation - so-called spinodal decomposition [39] else phase separation at liquation process. In S0, we have observed a change of slope in the curve $I(Q)$ near $Q=0.02 \AA^{-1}$ only (Fig. 2A). According to calculation, the maximum size of inhomogeneities in glass is about $15 \mathrm{~nm}$ (Fig. 3 - black squares), the volume fraction of pores is $1 \%$ of the total volume. The pair correlation function for S0 (Fig. 3) can be consider as a distribution of channel diameters which would be formed in this glass after leaching. I(Q) in the diapason $0.02-0.11 \AA^{-1}$ obeys the power law $Q^{-\alpha}$ with $\alpha=3.25$ (Fig. 2A - red straight line): it corresponds to scattering from highly rough surfaces (surface fractals) due to the presence of secondary silicates into the channels in this glass forming at heat treatment (liquation process). Thus we can conclude that the $\mathrm{S} 0$ glass is practically non porous.

\subsection{NCM In + PG7 and In+PG-MAP (S1 and S2 samples)}

Porod's law for In+PG7 (Fig. 2B) and In+PG-MAP (Fig.2D) at $Q>0.04 \AA^{-1}$ is performed clearly and this results in scattering on smooth channel surfaces. In the $\mathrm{S} 1$ sample at $Q<0.04 \AA^{-1}$, the scattering extends to the Guinier's region. In general, the picture looks like this: indium fills only a part of the total porous space in this sample, but a part of the pores remains empty (Table 2), that gives the Porod's law at large $Q$. It is necessary to note that the maximum cluster size (inhomogeneities, i.e. empty pores + metal, in the S1 sample) increases up to $20 \mathrm{~nm}$ (Fig. 3 red squares). The position of maximum corresponds to $\sim 8 \mathrm{~nm}$, which is clearly smaller than the diffraction size ( 11-15 nm) of indium nanoparticles obtained from analysis of diffraction patterns [4] for this NCM.

For NCM In+PG-MAP we have observed the crossover point at $Q=0.04 \AA^{-1}$ at which the slope (parameter $\alpha$ ) of the dependence $I(Q) \sim Q^{-\alpha}$ changes from 4.02 to 3.19 (red straights on Fig. 2D). This $Q$ value corresponds to a space scale $d=2 \pi / Q=15-16 \mathrm{~nm}$. The value $\alpha=3.19$ shows that in this NCM in porous space, the surface fractal structures exist on a spatial scale greater than $16 \mathrm{~nm}$. In this case, the maximum size of inhomogeneities in this NCM increases up to $60-70 \mathrm{~nm}$ (Fig. 3 - blue squares). 


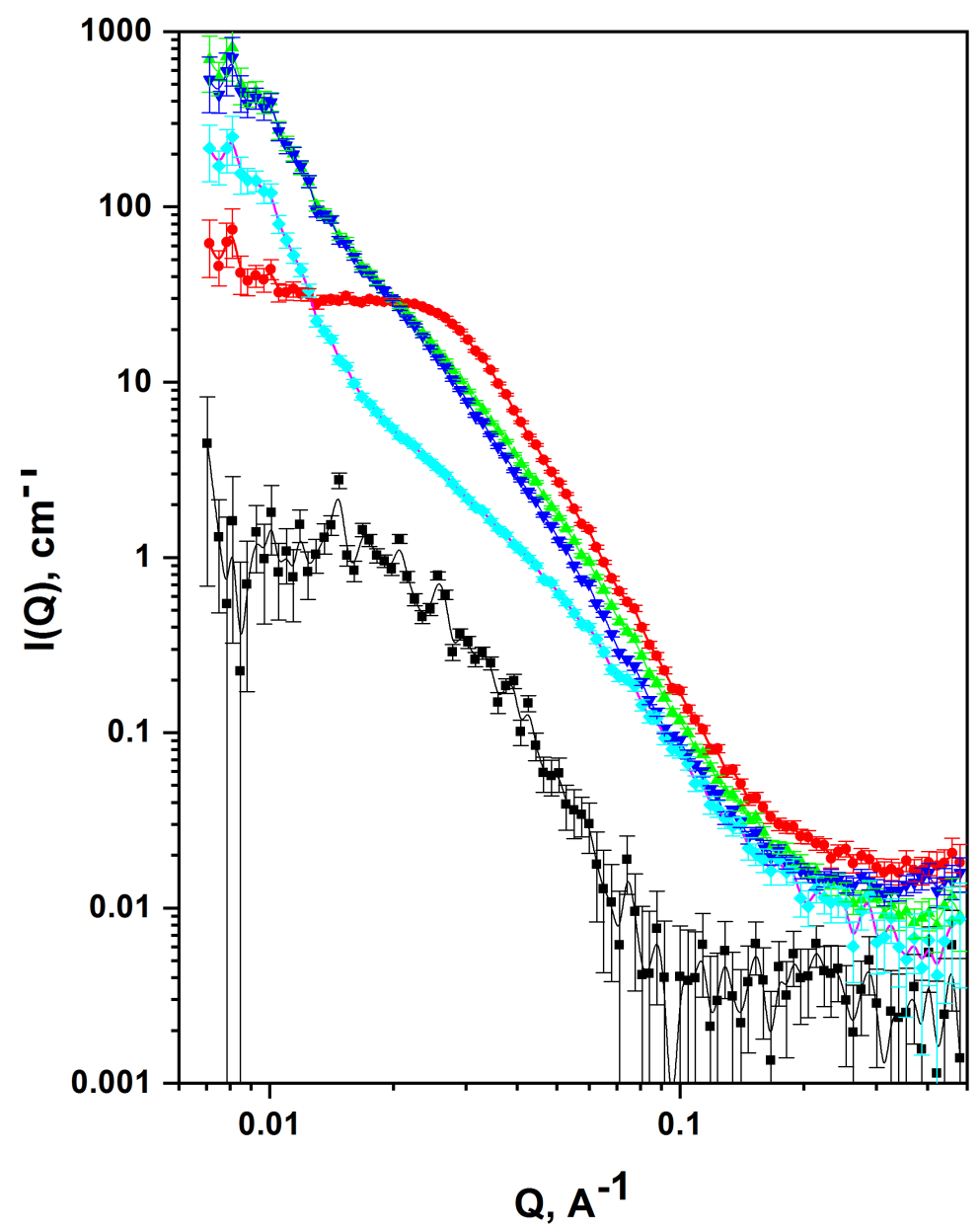

FIG. 1. Small-angle neutron scattering from samples: S0 - black squares, S1 (In + PG7) - red rounds, S2 (In+PG-MAP) - blue triangles, S3 (Sn+PG-MAP) - green triangles, S4 (Pb+PG-MAP) cyan rhombs

TABLE 2. Volume fractions of metals $\left(\varphi_{\text {Met }}\right)$ and voids $\left(\varphi_{\text {Pores }}\right)$ in samples $\mathrm{S} 0-\mathrm{S} 4$ obtained from the equations (6-9)

\begin{tabular}{|c|c|c|c|c|c|}
\hline Sample & S0 & S1 (In+PG7) & S2 (In+PG-MAP) & S3 (Sn+PG-MAP) & S4 (Pb+PG-MAP) \\
\hline$\varphi_{\text {Met }}, \%$ & 0 & 12.8 & 12.5 & 8.9 & 19.1 \\
\hline$\varphi_{\text {Pores }}, \%$ & 1.0 & 8.6 & 7.2 & 10.8 & 4.4 \\
\hline$\varphi_{\mathrm{Gl}}, \%$ & 99.0 & 78.6 & 80.3 & 80.3 & 76.4 \\
\hline
\end{tabular}

\subsection{NCM Sn+PG-MAP (S3 sample)}

For NCM Sn+PG-MAP we have also observed the crossover point at $Q=0.04 \AA^{-1}$ (as in for NCM In+PGMAP), but at this case, the parameter $\alpha$ in the dependence $I(Q) \sim Q^{-\alpha}$ changes from 4.06 to 2.92 (red straights on Fig. 2C). The value $\alpha=2.92$ shows that in this NCM in porous space the mass fractal structures exist on a spatial scale greater than $16 \mathrm{~nm}$. The maximum size of inhomogeneities in this NCM also increases to $60-70 \mathrm{~nm}$ (Fig. $3-$ green squares). 


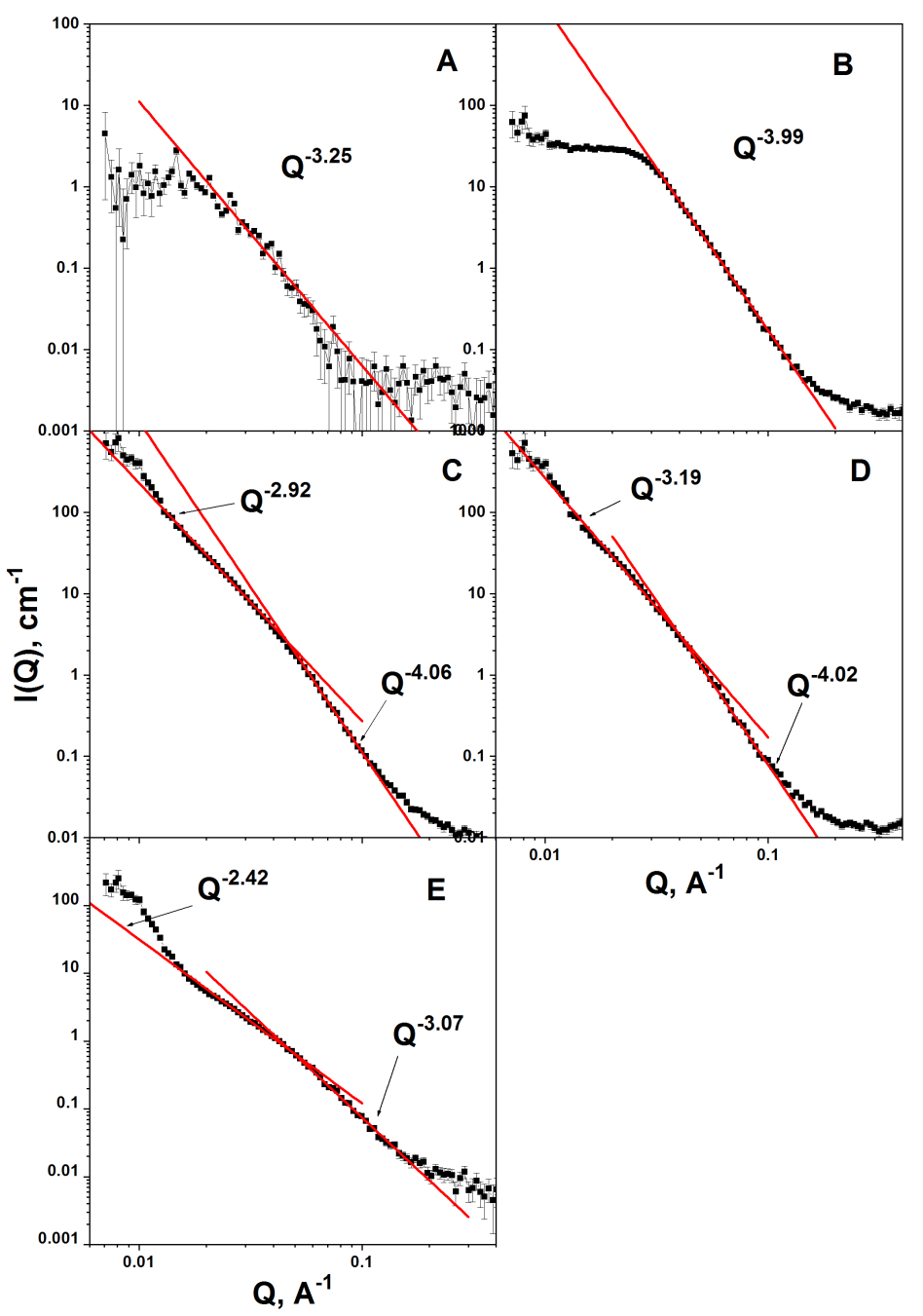

FIG. 2. SANS data and fractal characteristics of the samples S0-S4: A-S0, B - S1, C-S3, D $\mathrm{S} 2, \mathrm{E}-\mathrm{S} 4$

\subsection{NCM Pb+PG-MAP (S4 sample)}

The scattering laws differ essentially from scattering on In+PG-MAP and Sn+PG-MAP (Fig. 2E). For the region $Q>0.04 \AA^{-1} I(Q)$ follows as $\sim Q^{-3.07}$, that is typical for a strongly rough surface. The intersection of the background constant with the scattering curve at $Q=0.2 \AA^{-1}$ gives an approximate minimum roughness size of the order $d \sim 3 \mathrm{~nm}$. Since the scattering density of lead is approximately the same as for glass itself (Table 1), the picture of scattering is similar to the glass before leaching, when there are a lot of secondary silica into the pore space. Starting from $Q<0.04 \AA^{-1}$, the scattering is described as $I(Q) \sim Q^{-2.42}$ - which corresponds to scattering from mass fractals - polydisperse distribution of lead nanoparticles and empty pores with air. The volume fraction of free pores is significantly less than for other MAP samples (Table 2). NCM Pb+PG-MAP shows a typical correlation peak for alkali borosilicate glasses, but it is not at $Q=0.015 \AA^{-1}$, but at a position less than $Q<0.01 \AA^{-1}$. These peaks are also faintly visible on the samples starting from the second one. This is in agreement with the fact that if the cluster size for samples on base of MAP glasses is on the order of $d \sim 60-70 \mathrm{~nm}$, then the position of the correlation peak $Q_{\text {corr }} \sim 2 \pi / d$ should be in the range from 0.0089 to $0.01 \AA^{-1}$.

\subsection{Summary remarks}

For NCM In+PG7, In+PG-MAP and Sn+PG-MAP at $Q>0.04 \AA^{-1}$, we have observed Porod ‘s law for scattering intensity that corresponds to a smooth surface. It means that in this $Q$ region in the samples $\mathrm{S} 1, \mathrm{~S} 2$ and $\mathrm{S} 3$ the interface "matrix-air", having the greatest contrast $(\Delta \rho)^{2}$ among others (Table 1), plays the principle role. In the case of the $\mathrm{S} 4$ ( $\mathrm{Pb}+\mathrm{PG}-\mathrm{MAP})$ sample, the situation changes drastically: parameter $\alpha$ becomes equal to 3.07 at large $Q$, i.e. here, 


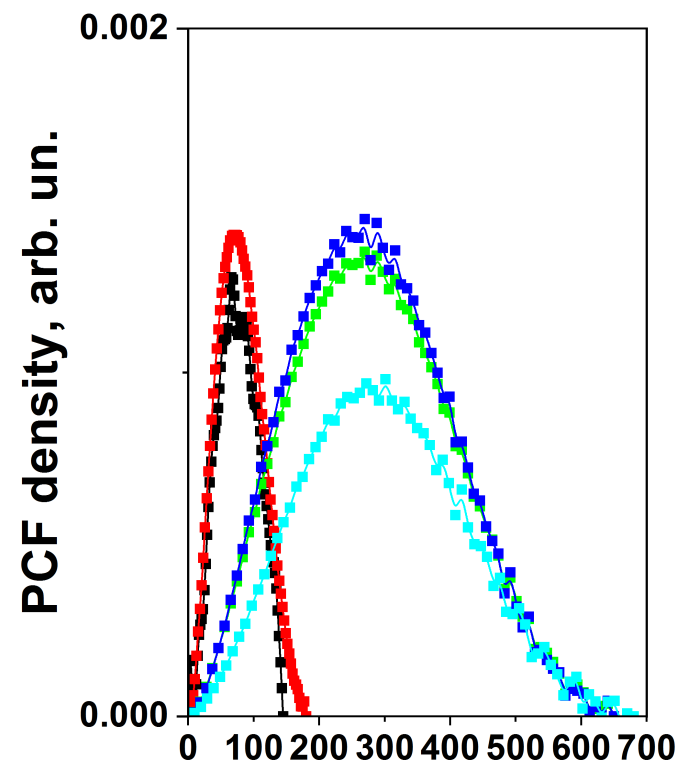

\section{Correlation length, A}

FIG. 3. Pair correlation functions (PCF) obtained after treatment by GNOM program (ATSAS package [33]) for samples: S0 -black squares, S1 - red squares, S2 - blue squares, S3 - green squares, S4 - cyan squares

we are dealing with a surface fractal structure mainly formed by lead atoms. Apparently, this is due to a significant difference in the surface tension coefficients $(\sigma)$ of these metals, since the filling takes place from the melt, followed by cooling and crystallization in the pores: indeed $\sigma_{\mathrm{Pb}}=440 \cdot 10^{-3} \mathrm{n} / \mathrm{m}, \sigma_{\mathrm{In}}=556 \cdot 10^{-3} \mathrm{n} / \mathrm{m}, \sigma_{\mathrm{Sn}}=530 \cdot 10^{-3} \mathrm{n} / \mathrm{m}[40]$. It looks like as, if a part of lead does not have time to shrink into large nanoparticles on cooling and at crystallization and becomes "smeared" in the matrix channels. Naturally, this process also takes place for indium and tin, but to a lesser extent due to the difference in surface tensions and the lower total pore filling (Table 2).

At $Q<0.04 \AA^{-1}$ in NCM Pb+PG-MAP and $\mathrm{Sn}+\mathrm{PG}-\mathrm{MAP}$ the parameters $\alpha$ are in the range from 2 to 3 , which correspond to the mass fractal; however, for indium, this parameter $(\alpha=3.19)$ indicates the formation of a surface fractal structure. Therefore, in wide-pore glass with the usual cooling mode of NCM. It is most likely, that this is due to the low melting point of indium: i.e. in the wide-pore glass at the usual cooling mode of NCM, some of the indium (similar to lead) does not have time to completely collect into nanoparticles and remains in a "smeared" state over the porous space. In the case of In+PG7, the pore diameter is essentially smaller, and indium has time to crystallize into nanoparticles to a greater extent.

Now let us discuss the results of pair correlation function (PCF) analysis presented in Fig. 3. As it has been shown in the paper [4] in NCM In+PG7 the nanoparticles have anisotropic form with two characteristic diffraction sizes: 11 and $15 \mathrm{~nm}$. However, it can be seen from Fig. 3 that the value of the correlation length is in the range $0-17 \mathrm{~nm}$, i.e. in the NCM there is a significant part of indium agglomerates much less than the diffraction size. On the other hand, we have not seen such small particles in the diffraction spectra. This disagreement is easy to explain. In diffraction studies, we have a response from a crystal structure only, since scattering from the amorphous metal phase "sinks" in the general strong diffuse background from the matrix. In SANS measurements, only a contrast $\Delta \rho$ plays the principle role. The contrast for the crystalline and amorphous phases is the same. Thus, we can conclude that this NCM contains a considerable portion of indium in the amorphous state. A similar argumentation one can do for the samples S2 - S4, only in these samples, according to preliminary diffraction measurements, the diffraction size of nanoparticles is in the range of $25-40 \mathrm{~nm}$.

There is one more argument in favor of the multicomponent structure of metals in these NCMs. For similar random dendrite 3D systems (as our glasses), it has been found that the percolation threshold $\rho_{c}$ is equal to 0.2 [41]. As it is seen from Table 2, the total amount of embedded metals in all cases (excepting Pb+PG-MAP) is smaller than $\rho_{c}$, but a through conduction has been observed for all samples S1 - S4 [15, 18-20,42]. This means that the spatial organization of the internal structure of embedded metals can be described as follows: there are sufficiently large 
crystalline nanoparticles that either border directly on each other or are linked by weak bonds, which, apparently, are largely consisted of amorphous metals.

To clarify the situation and to obtain estimations of the content of the crystalline and amorphous phases, we are going to carry out additional combined diffraction and SANS studies of the temperature evolution of diffuse scattering in vicinities of principle Bragg peaks and $\alpha$ parameters in the $Q^{-\alpha}$ dependence at melting and crystallization of metals in these NCM.

\section{Conclusion}

The fractal characteristics of NCM on base of porous alkali borosilicate glasses containing the low-melting metals (In, $\mathrm{Sn}$ and $\mathrm{Pb}$ ) have been obtained from analysis of SANS data at room temperature. It is shown, that for all NCM there is the critical space scale near $Q=0.04 \AA^{-1}(15-16 \mathrm{~nm})$, at which one can see a crossover in the behavior of the scattering intensity $I(Q) \sim Q^{-\alpha}$.

It is shown that in these NCM we are dealing with a complex system that combines both the fractal properties of embedded metals and the matrix itself. Combined analysis of SANS spectra, pair correlation function and conventional diffraction patterns permits to conclude that the crystalline and amorphous phases of embedded metals coexist in these NCM.

\section{Acknowledgements}

A. A. Naberezhnov thanks the RFBR-BRICS grant 19-52-80019 for partial financial support. A. V. Fokin thanks the Russian Foundation for Basic Research, grant 19-02-00760. In FLNP JINR this work was partly supported by RF Ministry of Education and Science, grant number 19-52-44003 \19.

\section{Conflict interests}

Authors declare that they have no conflict interests.

\section{References}

[1] Lee M.K., Tien C., et al. Structural variations in nanosized confined gallium. Physics Letters A, 2010, 374, P. $1570-1573$.

[2] Tanaka M., Takeguchi M., Furuya K. In situ observation of indium nanoparticles deposited on Si thin films by ultrahigh vacuum field emission transmission electron microscope. Surface Science, 1999, 433-435, P. 491-495.

[3] Balamurugan B., Kruis F.E., et al. Size-induced stability and structural transition in monodispersed indium nanoparticles. Appl. Phys. Lett., $2005,86,083102$.

[4] Naberezhnov A.A., Sovestnov A.E., Fokin A.V. Crystal Structure of Indium and Lead under Confined Geometry Conditions. Technical Physics, 2011, 56 (5), P. 637-641.

[5] Bonetti E., Pasquini L., et al. Vibrational density of states of nanocrystalline iron and nickel. Jour. Appl. Phys., 2000,88 (8), P. $4571-4575$.

[6] Trampenau J., Bauszuz K., Petry W., Herr U. Vibrational behaviour of nanocrystalline Ni. Nanostruct. Mater., 1995 , 6, P. 551-554.

[7] Fultz B., Robertson J.L., et al. Phonon density of states of nanocrystalline Fe prepared by high-energy ball milling. J. Appl. Phys., 1996, 79, P. 8318-8322.

[8] Suck J.B. Metallic Nanocrystals and Their Dynamical Properties. In: Gemming S., Schreiber M., Suck JB. (eds) Materials for Tomorrow. Springer Series in Materials Science, 2007, 93. Springer, Berlin, Heidelberg, 196 p.

[9] Parshin P.P., Zemlyanov M.G., et al. Atomic Dynamics of Lead Introduced into Nanopores in Glass. JETP, 2011 , 111 (6), P. $996-1002$.

[10] Parshin P.P., Zemlyanov M.G., et al. Atomic Dynamics of Tin Nanoparticles Embedded into Porous Glass. JETP, 2012 , 114 (3), P. $440-450$.

[11] Borman V.D., Pushkin M.A., Tronin V.N., Troyan V.I. Evolution of the electronic properties of transition metal nanoclusters on graphite surface. JETP, 2010, 110 (6), P. 1005-1025.

[12] Sovestnov A.E., Naberezhnov A.A., et al. Study of Palladium Nanoparticles Synthesized in Alkali Borosilicate Glass Pores by the X-Ray Line Shift Method. Physics of the Solid State, 2013, 55 (4), P. 837-841.

[13] Balamurugana B., Maruyama T. Size-modified d bands and associated interband absorption of Ag nanoparticles. Jour. Appl. Phys., 2007, 102, 034306.

[14] Rao C.N.R., Kulkarni G.U., et al. Metal nanoparticles, nanowires, and carbon nanotubes. Pure Appl. Chem., 2000,72 (1-2), P. $21-33$.

[15] Kumzerov Yu.A., Naberezhnov A.A. Effect of restricted geometry on superconducting properties of low-melting metals (Review). Low Temperature Physics, 2016, 42 (11), P. 1028-1040.

[16] Tien C., Wur C.S., et al. Double-step resistive superconducting transitions of indium and gallium in porous glass. Phys. Rev. B, 2000, 61 (21), P. 14833-14838.

[17] Charnaya E.V., Tien C., Lee M.K., Kumzerov Yu.A. Superconductivity and structure of gallium under nanoconfinement. J. Phys.: Condens. Matter, 2009, 21, 455304.

[18] Tien C., Charnaya E.V., et al. Vortex avalanches in a Pb-porous glass nanocomposite. Phys. Rev. B, $2011, \mathbf{8 3}, 014502$.

[19] Panova G.Kh., Nikonov A.A., Naberezhnov A.A., Fokin A.V. Resistance and Magnetic Susceptibility of Superconducting Lead Embedded in Nanopores of Glass. Physics of the Solid State, 2009, 51 (11), P. 2225-2229.

[20] Shikov A.A., Zemlyanov M.G., et al. Superconducting Properties of Tin Embedded in Nanometer-Sized Pores of Glass. Physics of the Solid State, 2012, 54 (12), P. 2345-2350.

[21] Enke D., Janowski F., Schwieger W. Porous glasses in the 21st century - a short review. Microporous and Mesoporous Materials, 2003, 60, P. 19-30. 
[22] Haller W. In Solid Phase Biochemistry, Scouten W.H. (Ed.), Wiley, New York, 1983, 535 p.

[23] Kumzerov Yu.A., Nabereznov A.A., Savenko B.N., Vakhrushev S.B. Freezing and melting of mercury in porous glass. Phys. Rev. B, 1995, 52 (7), P. 4772-4774.

[24] Golosovsky I.V., Delaplane R.G., Naberezhnov A.A., Kumzerov Yu.A. Thermal motions in lead confined within porous glass. Phys. Rev. B, 2004, 69, 132301.

[25] Kibalin Y.A., Golosovsky I.V., et al. Neutron diffraction study of gallium nanostructured within a porous glass. Phys. Rev. B, $2012,86,024302$.

[26] Ostanevich Yu.M. Timeofflight smallangle scattering spectrometers on pulsed neutron sources. Makromol. Chem. Macromol. Symp., 1988, 15, P. 91-103.

[27] Kuklin A.I., Islamov A.Kh., Gordeliy V.I. Two-detector System for Small-Angle Neutron Scattering Instrument. Neutron News, 2005, 16, P. 16-18.

[28] Soloviev A.G., Solovieva T.M., et al. SAS. The package for Small-Angle Neutron Scattering Data Treatment. Version 2.4. Long Write-Up and User's Guide. JINR Communication, 2003, P10-2003-86, 24 p.

[29] Soloviev A.G., Solovjeva T.M., et al. SAS program for two-detector system: seamless curve from both detectors. Journal of Physics: Conf. Series, 2017, 848 (1), 012020.

[30] Bale H.D., Schmidt P.W. Small-Angle X-Ray-Scattering Investigation of Submicroscopic Porosity with Fractal Properties. Phys. Rev. Lett., 1984, 53, P. 596-599.

[31] Teixeira J. Small-angle scattering by fractal systems. J. Appl. Crystallogr., 1988, 21, P. 781-785.

[32] Neutron/X-ray Scattering Length Density Calculator. URL: https://sld-calculator.appspot.com.

[33] Franke D., Petoukhov M.V., et al. ATSAS 2.8: a comprehensive data analysis suite for small-angle scattering from macromolecular solutions. J. Appl. Cryst., 2017, 50, P. 1212-1225.

[34] Feigin L.A., Svergun D.I. Structure Analysis by Small Angle X Ray and Neutron Scattering, Princeton, New Jersey, 1987,335 p.

[35] Levitz P., Ehret G., Sinha S.K., Drake J.M. Porous vycor glass: The microstructure as probed by electron microscopy, direct energy transfer, smallangle scattering, and molecular adsorption. Jour. Chem. Phys., 1991, 95, 6151.

[36] Naberezhnov A.A., Vakhrushev S.B., Okuneva N.M., Sysoeva A.A. SANS studies of nanocomposites Sodium nitrite + Porous glasses. Ferroelectrics, 2019, 539 (1), P. 16-21.

[37] Höhr A., Neumann H.-B., et al. Fractal surface and cluster structure of controlled-pore glasses and Vycor porous glass as revealed by smallangle X-ray and neutron scattering. Phys. Rev. B, 1988, 38 (2), P. 1462-1467.

[38] Wiltzius P., Bates F.S., Dierker S.B., Wignall G.D. Structure of porous Vycor glass. Phys. Rev A Gen. Phys., 1987,36 (6), 2991.

[39] Cahn J.W. On spinodal decomposition. Acta Metall., 1961, 9 (9), 795.

[40] Surface tension of metals in liquid state. URL: https://tehtab.ru/Guide/GuidePhysics/SurfaceTension/ SurfaceTensionofLiquidMetall [In Russian]

[41] Grannan D.M., Carland J.C., Tanner D.B. Critical Behavior of the Dielectric Constant of a Random Composite near the Percolation Threshold. Phys. Rev. Lett., 1981, 46 (5), P. 375-378.

[42] Tien C., Wur C.S., et al. Double-step resistive superconducting transitions of indium and gallium in porous glass. Phys. Rev. B, 2000, 61 (21), P. 14833-14838. 